\title{
High Temperature Vibratory Response of Hastelloy-X: Stereo-DIC Measurements and Image Decomposition Analysis
}

\author{
R.B. Berke ${ }^{1,2}$ - C.M. Sebastian ${ }^{3}$ - R. Chona ${ }^{4}$ - E.A. Patterson ${ }^{3}$ - J. Lambros ${ }^{1}$
}

Received: 26 April 2015 / Accepted: 3 September 2015 /Published online: 17 September 2015

(C) The Author(s) 2015. This article is published with open access at Springerlink.com

\begin{abstract}
The mechanical behavior of solids in combined high-temperature and vibratory environments, such as those experienced during hypersonic flight, are historically not well explored. In this work on Hastelloy-X plates, elevated temperatures were achieved by induction heating and periodic vibratory loading was applied using a shaker. Surface displacements and strains were measured using stereo digital image correlation (DIC) in the blue spectrum to alleviate issues associated with thermal radiation. Through the use of image decomposition techniques the resultant high-quality experimental data were used to validate numerical simulations of combined thermoacoustic loading. The simulations were based on the deformed shape and the corresponding temperature distributions measured experimentally as well as taking into account the thermal dependence of Hastelloy-X mechanical properties.
\end{abstract}

Keywords Stereo digital image correlation · High temperature measurement · Thermo-acoustic loading · Induction heating · Image decomposition analysis

R.B. Berke

ryan.berke@usu.edu

1 Department of Aerospace Engineering, University of Illinois, Urbana-Champaign, 306 Talbot Lab, Urbana, IL 61801, USA

2 Present address: Department of Mechanical and Aerospace Engineering, Utah State University, 4130 Old Main Hill, Logan, UT 84322, USA

3 School of Engineering, University of Liverpool, The Quadrangle, Brownlow Hill, Liverpool L69 3GH, UK

4 Structural Sciences Center, U.S. Air Force Research Laboratory, Dayton, OH 45433, USA

\section{Introduction}

Digital image correlation (DIC) is a versatile measurement technique, capable of obtaining full-field displacements and strains at a range of length scales, temperatures, and loading rates. Motivated by the increasing interest in applications involving significant thermal effects, such as aero-engines, spacecraft re-entry, aircraft hypersonic flight, and nuclear power applications, recent efforts have extended the upper temperature limit of the DIC technique by the use of optical bandpass filters and narrow band lighting, which reduces the influence of light radiated by the specimen at high temperatures [1-6]. Grant et al. demonstrated this approach for hightemperature DIC by using blue light along with blue range bandpass filters to measure the coefficient of thermal expansion of RR1000 (a nickel-based alloy) at temperatures up to $1000{ }^{\circ} \mathrm{C}$ with two-dimensional (2D)-DIC [1]. Using a similar method, Novak and Zok demonstrated the blue-filtering technique on a $\mathrm{C} / \mathrm{SiC}$ composite at temperatures up to $1500{ }^{\circ} \mathrm{C}$ [2]. Later, Chen et al. and Pan et al. applied the blue-filtering technique to stereo-DIC - in which two cameras are used to make three-dimensional (3D) measurements - at temperatures up to 1100 and $1200{ }^{\circ} \mathrm{C}$, respectively [3, 4]. Most recently, Berke and Lambros showed that the temperature range can be extended even further by using ultraviolet lighting and optics, which operate at an even shorter wavelength than blue light [6].

However, in many of the high temperatures applications mentioned above (aero-engines, spacecraft re-entry, hypersonic flight) it is the coupled effects of thermal and mechanical loading, often at high frequencies, that provide an extreme operating environment which can critically affect structural performance and cause failure by the interaction of coupled, non-linear, failure modes that are otherwise not present [7]. Specifically, the combined high-temperature and vibration 
environment, as is experienced by aircraft during hypersonic flight and which is of interest to this study, is historically not well explored. It is only recently that dynamic experiments have been attempted at high temperature. Abotula et al. used a pair of high-speed cameras to capture the deformation of Hastelloy (a nickel-based superalloy) samples at temperatures up to $900{ }^{\circ} \mathrm{C}$ under shock wave loading [8]. To illuminate their specimens, Abotula et al. [8] used a $450 \mathrm{~nm}$ filter with a full width at half maximum (FWHM) of $40 \mathrm{~nm}$ and a transmission efficiency of $45 \%$, which significantly reduced the amount of light reaching the camera sensors - potentially a critical drawback in high-speed imaging applications which require limited image exposure times. To overcome this limit, they used a high energy flash lamp which delivered $220 \mathrm{~kW}$ to the specimen for $5 \mathrm{~ms}$. In another high-speed study, Vautrot et al. used a 500 frames per second (fps) camera to study the plastic deformation of high-carbon steels at up to $720^{\circ} \mathrm{C}$ under strain rates ranging between $10^{-3} / \mathrm{s}$ to $400 / \mathrm{s}$ using a split Hopkinson pressure bar [9]. They used a much broader filter which passed the entire $400-700 \mathrm{~nm}$ visible wavelength range while screening out infrared, and were able to conduct their tests using a lighting system of white light LEDs.

Thus it is clear that although there is an increasing need to characterize materials and structures under extreme combined thermal and mechanical loading environments, there is a lack of such experimental studies. Consequently, thermomechanically coupled phenomena are not well understood and predictive models in these extreme environments are sometimes inaccurate. The present study seeks to address the lack of such experimental data and aims to,

(i) develop procedures to obtain high quality experimental data suitable for validation of numerical results in a combined thermal and mechanical/vibratory environment,

(ii) study the influence of elevated temperature on the vibratory response of a metallic plate, and specifically investigate the (potential) change of resonant modes/ frequencies at elevated temperatures above room temperature, and

(iii) quantify the (potential) discrepancies between full-field experimental measurements and their counterpart numerical simulations.

To achieve these goals, thermo-vibratory loading conditions are combined on a thin plate of a nickel-based superalloy using magnetic induction for heating and a mechanical shaker for resonant vibratory loading. Combined high-temperature/ vibration experimental measurements are based on the optical technique of stereo-DIC that measures the full-field surface displacements and strains of the plate. A companion computational model is also used to perform a modal analysis by assuming an elastic plate but allowing for the variation in elastic properties with temperature. Resonant modes are then compared between experiments and modeling using an image decomposition technique involving 2D Tchebichef polynomials.

\section{Experimental Methods}

Experiments were conducted on $120 \times 80 \times 1 \mathrm{~mm}$ plates of Hastelloy-X, a nickel-based superalloy, purchased from American Special Metals Inc. [Miami, FL, USA]. A 5 mm diameter hole was drilled through the center of the plate for attaching one end of a threaded stinger rod to the plate while attaching the other end to a mechanical shaker. The front surface of the plate was speckled using high temperature refractory paints purchased from Aremco [Valley Cottage, NY, USA]. To help the paints adhere, the plate's surface was preoxidized in a box furnace by heating the specimen to $800^{\circ} \mathrm{C}$ (i.e. higher than the expected maximum in the experiments and below the heat treatment temperature during manufacture) and maintaining the temperature for a dwell time of about $20 \mathrm{~min}$ before cooling. The now-oxidized front surface was then speckled white with Pyropaint 634-ZO, which has a maximum temperature rating of $1800{ }^{\circ} \mathrm{C}$. The white paint was cured at room temperature for $2 \mathrm{~h}$ and at $100{ }^{\circ} \mathrm{C}$ for $2 \mathrm{~h}$ as described in the manufacturer's curing instructions. The surface was then additionally speckled with a random black speckle pattern, to provide contrast, using Hi-E Coat 840-CM, which has a maximum temperature rating of $1371{ }^{\circ} \mathrm{C}$. The black paint was cured for $1 \mathrm{~h}$ at room temperature, followed by $30 \mathrm{~min}$ at $100{ }^{\circ} \mathrm{C}$ and $1 \mathrm{~h}$ at $260{ }^{\circ} \mathrm{C}$ as described by the manufacturer's instructions. The heating and cooling rates for all thermal cycles during paint application were about $20{ }^{\circ} \mathrm{C} / \mathrm{min}$.

The center of the plate was secured to one end of the threaded stinger rod by tightening a pair of nuts at the front and back of the plate. The stinger provided the sole support for the plate (i.e., there were no strings or wires attached to the plate). An image of the speckled surface is provided in Fig. 1, with the nut visible in the center of the plate. The other end of the rod was attached to a mechanical shaker which was used to vibrate the plate in a direction perpendicular to the plate's surface (i.e., along the length of the stinger). A schematic of the experimental apparatus which includes the plate/rod/shaker assembly is given in Fig. 2.

The plate was heated by magnetic induction using a pancake-type coil [10]. The coil was positioned behind the plate so as not to obstruct image capture of the front surface of the plate. An opening was included in the center of the coil for the stinger rod to connect to the specimen without either the rod or the specimen coming into contact with the coil. The temperature was monitored using a single $\mathrm{K}$ type thermocouple bonded to the back of the plate at the position where the threaded rod was attached. Like the 


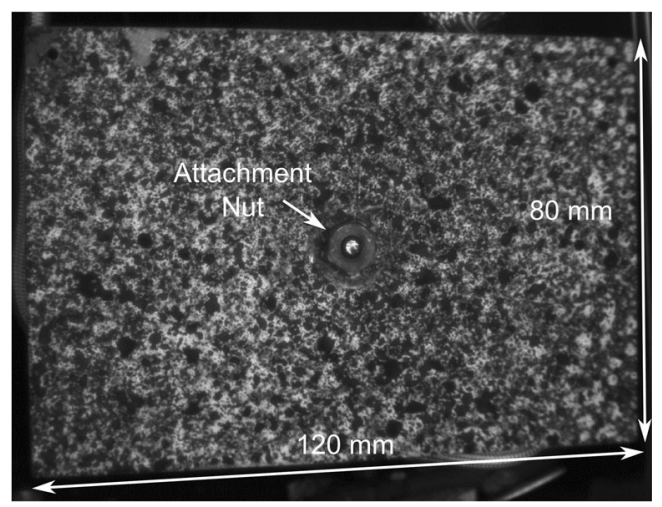

Fig. 1 The speckled surface of the $120 \times 80 \times 1 \mathrm{~mm}$ plate specimen, with the attachment nut visible in the center of the plate

rod, the thermocouple wire also passed through the center of the coil without contacting the coil.

Heating of the initially nominally flat plate by the induction coil was heterogeneous both in plane and through-thickness, resulting in significant out-of-plane deformation. In addition, the particular temperature distribution, and consequently the resulting out-of-plane displacement, is unique to each plate tested since placement and geometry of coil and sample cannot guarantee identical conditions each time. Therefore both had to be measured at the start of each experiment. The shape of the plate was measured from stereo image pairs collected before and after heating using Vic-3D, a commercially available stereo-DIC algorithm from Correlated Solutions Inc. [Columbia, SC, USA]. A typical set of measurements for the front surface of the plate before and after heating are plotted in Fig. 3. This measurement determined the starting geometry prior to the application of vibratory loading. Note that in any

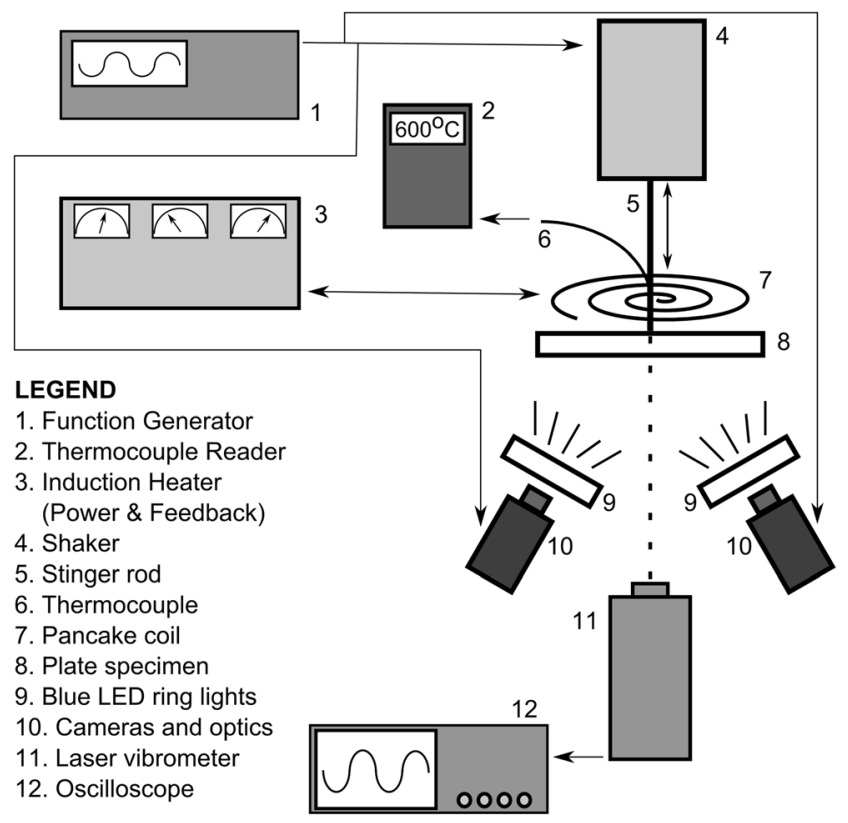

Fig. 2 Schematic of experimental setup realistic application of a non-uniformly heated thin plate, the starting state prior to vibratory loading will be a curved shape (Fig. 3(b)), even if the initial room temperature configuration is flat. However, note also that there is some small curvature even in the nominally flat plate at room temperature (Fig. 3(a)) although it is a order of magnitude less than for the heated plate (note different scales in Fig. 3(a) and (b)).

The measured deformed shape was used to estimate the inplane temperature distribution across the front surface of the plate. This was accomplished by (1) taking the static images of the speckled surface before and after heating, (2) calculating the thermal strains from these images, and (3) dividing the thermal strains by the temperature-dependent coefficient of thermal expansion (CTE) of Hastelloy-X provided by the manufacturer [11] and which was assumed to be isotropic and constant through the thickness of the plate. The resultant estimated temperature distribution, which is shown in Fig. 4 for the curved plate from Fig. 3(b), is non-uniform due to the irregular shape and spacing of the induction coil. It has a maximum estimated temperature of around $600{ }^{\circ} \mathrm{C}$ occurring at the bottom edge of the plate, in this case. There is a hole in the center of the contour plot of Fig. 4 because of the exclusion of the attachment nut from the DIC calculations.

To apply vibration loads, a sine wave voltage was supplied to the shaker using a function generator (see Fig. 2, components 1 and 4). The out-of-plane displacement of the plate was monitored from the front using a laser vibrometer connected to an oscilloscope (Fig. 2, components 11 and 12). Resonant frequencies were then found by adjusting the sine wave over a range of frequencies until a local maximum in the amplitude of the displacement was observed. A total of 9 resonant frequencies were observed at room temperature, and another 9 were observed at high temperature. The form of the modal shapes implied that the same location could not be used to find all of the resonant frequencies. Most resonant modes were identified by monitoring the top left corner of the plate. However, modes 5 and 7 , which had near zero displacement at the corners, were identified by monitoring the middle of the left edge of the plate. In addition, the velocity data in Fig. 5 were obtained at the center of the plate by pointing the laser at the attachment nut once the resonant mode had been established in each case. Data shown in Fig. 5 are as-recorded raw data and some local spikes seen in some signals are likely from much higher frequency electrical noise and do not interfere with the measurement of the resonant frequency in each case.

For each of the resonant frequencies, a series of stereo images were taken using a pair of $1024 \times 1024$ pixel CCD cameras [Prosilica GX1050, Allied Vision Technologies $\mathrm{GmbH}$, Stradtroda, Germany] and $50 \mathrm{~mm}$ lenses with bluerange bandpass optical filters [BP470, Midwest Optical, Palatine, IL, USA] resulting in an average magnification of $0.13 \mathrm{~mm} / \mathrm{pixel}$. The cameras were triggered using the Fulcrum module of the Vic-Snap image acquisition software provided 


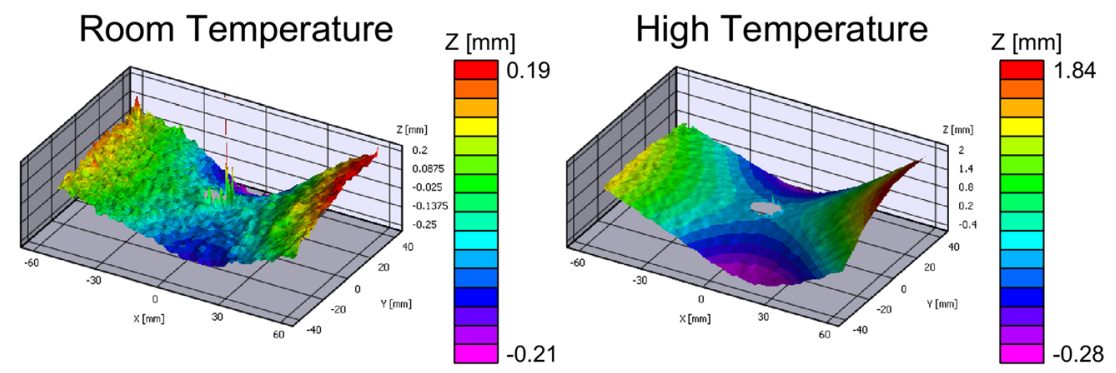

Fig. 3 Out-of-plane deformation (in mm) measured for the entire front surface of the plate using stereo DIC for the stationary plate at room temperature (left $)$ and elevated temperatures (right). Note that although there is some initial curvature prior to heating, the displacements due to non-uniform heating are an order of magnitude larger than the initial curved shape

by Correlated Solutions Inc. [Columbia, SC, USA]. To prevent motion blur, the blue LED ring lights were lit as brightly as possible and the exposure time for the cameras was set as low as possible. The minimum exposure time of the cameras was 10 microseconds. The maximum frame rate of the cameras was $112 \mathrm{fps}$, which was much slower than the oscillation of the plate. To correct for the slower frame rate, the Fulcrum software uses phase-locking to time image acquisition such that images taken from successive periods of oscillation can later be reconstructed into one representative period. Image pairs were acquired every $5^{\circ}$ in the period of the oscillation, resulting in a total of 71 image pairs for each resonant mode. Displacements and strains were computed using Vic-3D. The analysis was performed with a $29 \times 29$ pixel subset at an interval of 7 pixels, which were found to work satisfactorily based on rigid-body motion experiments of the specific patterns in each case. Pixel subsets which overlapped with the attachment nut were excluded from all DIC computations. These experimental parameters were chosen based on prior work in which detailed assessment of uncertainties of 3-dimensional dynamic measurements were made [12].

\section{Modal Analysis}

A modal analysis was performed to reproduce the experiments using commercially available finite element analysis (FEA) software [13]. Simulations were performed both at room

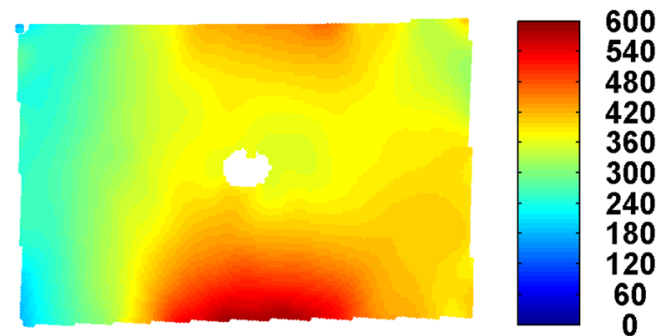

Fig. 4 Estimated temperature distribution (in ${ }^{\circ} \mathrm{C}$ ) on the entire front surface of the plate computed from the stereo DIC data. There is a hole at the center where the attachment nut was excluded from the DIC computation temperature and at high temperature. At both temperatures the initial shape of the plate as obtained from the DIC measurements, shown in Fig. 3, was used as the starting FEA model geometry. The effect of temperature on material properties was taken into account in the high-temperature simulation by assigning the elastic modulus of the material according to the estimated temperature distribution in Fig. 4. No thermal expansion or conduction was explicitly modeled in the high-temperature simulation and both simulations assumed a small strain linear elastic response (albeit with the inhomogeneous elastic modulus as described above for the high temperature case). Note that since the experimental data naturally cannot extend all the way to the edges of the plate, as the DIC measurements are made at the center pixel of subsets, a small amount of extrapolation, i.e. equivalent to approximately half a subset or $1.82 \mathrm{~mm}$, had to be performed to extend the estimated temperature distribution over the finite element (FE) mesh which did cover the entire area of the plate.

A $120 \times 80$ element FE mesh $(1 \mathrm{~mm}$ element size $)$ was created in the $x-y$ plane with the z-direction defined by the DIC measurements. For the high temperature simulation, the nodal temperatures were also obtained from the DIC measurement-based estimate described above, an example of which is illustrated in Fig. 4. Two-dimensional shell elements were used with a thickness of $1.016 \mathrm{~mm}$ corresponding to the measured values for the shape of the plate shown in Fig. 3. Poisson's ratio was assumed uniform and constant at 0.3 in both simulations. Since the elastic modulus decreases with increasing temperature, the value for each element was assigned depending on the local temperature and the temperature-dependent elastic modulus material properties for Hastelloy-X taken from the literature [11]. For this purpose, the estimated temperature map shown in Fig. 4 was divided into 6 different contour levels of $80^{\circ} \mathrm{C}$ each, resulting in the distribution shown in Fig. 6. The value (numbers shown in GPa in Fig. 6) of elastic modulus corresponding to the temperature range within each contour was then assigned to the corresponding elements. A custom Matlab script was used to write the node locations and element information to a text 
Fig. 5 Out-of-plane velocity measured for each mode at room temperature at the center of the plate using a laser vibrometer (vertical axis). Note that the time scale (horizontal axis) is different for each row
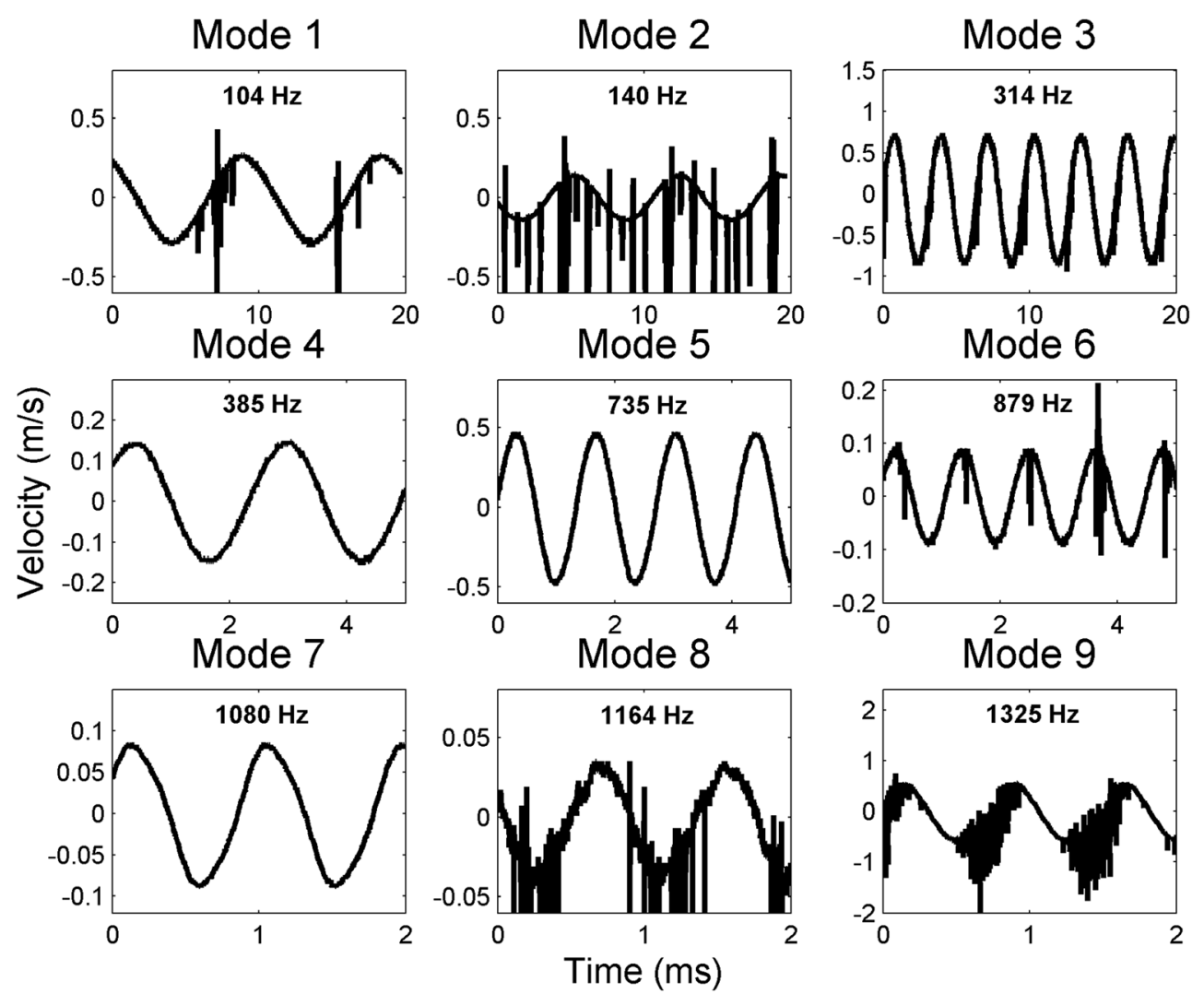

file in the format used by the FE solver such that it could be imported into the model.

\section{Results and Discussion}

\section{Modal Analyses}

The modal shapes measured by DIC at room temperature are plotted in Fig. 7 superimposed on the photograph of the undeformed plate in Fig. 1. Four specific locations are indicated with colored markers on each mode shape, representing the

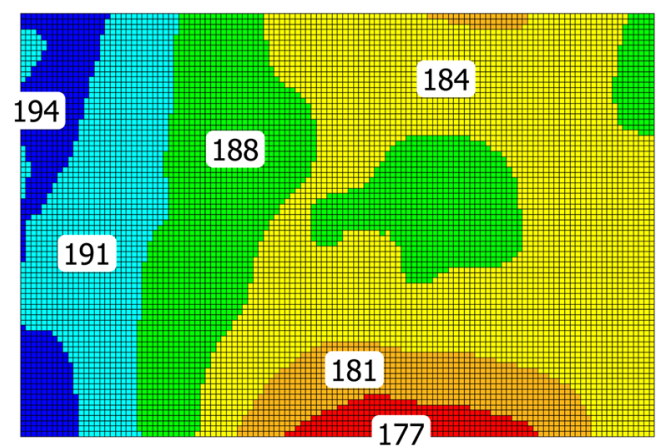

Fig. 6 FE mesh temperature distribution generated from Fig. 4 by dividing it into 6 contours. The elastic modulus values (in GPa) corresponding to each contour are shown overlaid in the figure positions of the maximum and minimum out-of-plane displacements in that mode shape. Note that in all of the modes, except 5 and 7, the maximum and minimum displacements occur at the corners of the plate. In modes 5 and 7 the corner displacements are near zero and the maximum and minimum displacements occur elsewhere on the edges of the plate in which case the specific points of observation are moved to those locations of observed extrema.

The displacements of each location marked in Fig. 7 are plotted throughout the period of oscillation in Fig. 8 using the same colors and style of markers as in Fig. 7. For each mode, Fig. 8 can be used to identify which locations oscillate inphase with each other and which oscillate out-of-phase. For example, Mode 1 consists of a rigid-body rotation about a vertical axis through the centerline of the plate, and Fig. 8 shows that for this mode the left two corners move in-phase with each other and the right two corners move in-phase with each other. Similarly, Mode 2 consists of a rigid-body rotation about an approximately horizontal axis, and Fig. 8 shows that the top two and bottom two corners move in-phase, respectively. Mode 3 in Fig. 7 corresponds to bending about a vertical axis, and therefore all four corners move in phase, as seen in Fig 8. Mode 4 corresponds to a warping component, with each pair of diagonally opposite corners moving in-phase (see Mode 4 in Fig. 8). Modes 5 through 9 each contain higher order combinations of flexural and rotational behavior, and similar conclusions can be drawn about the oscillation of the 
Fig. 7 Modal shapes obtained from stereo-DIC at room temperature, with the positions of the four locations plotted in Fig. 8 indicated by colored markers. Due to zero displacements in the corners of modes 5 and 7 , alternative locations have been selected instead
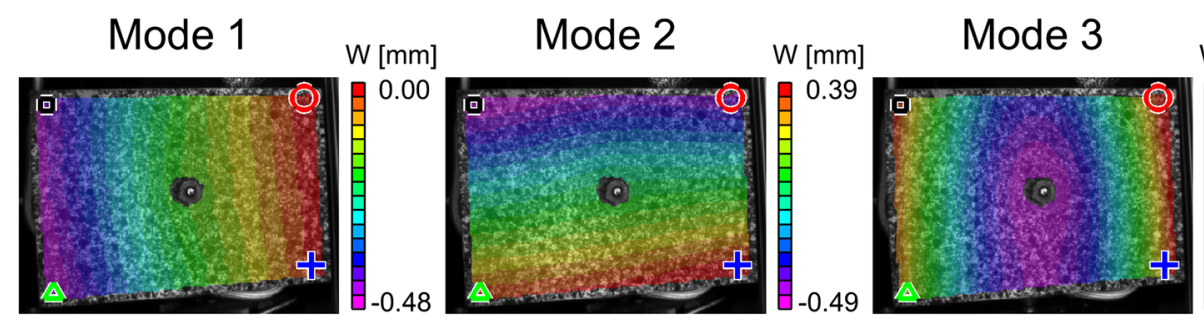

W [mm]
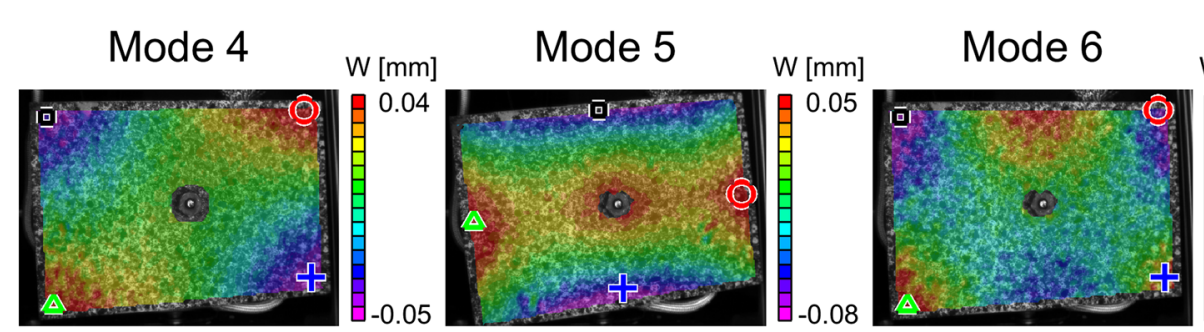

$\mathrm{W}[\mathrm{mm}]$
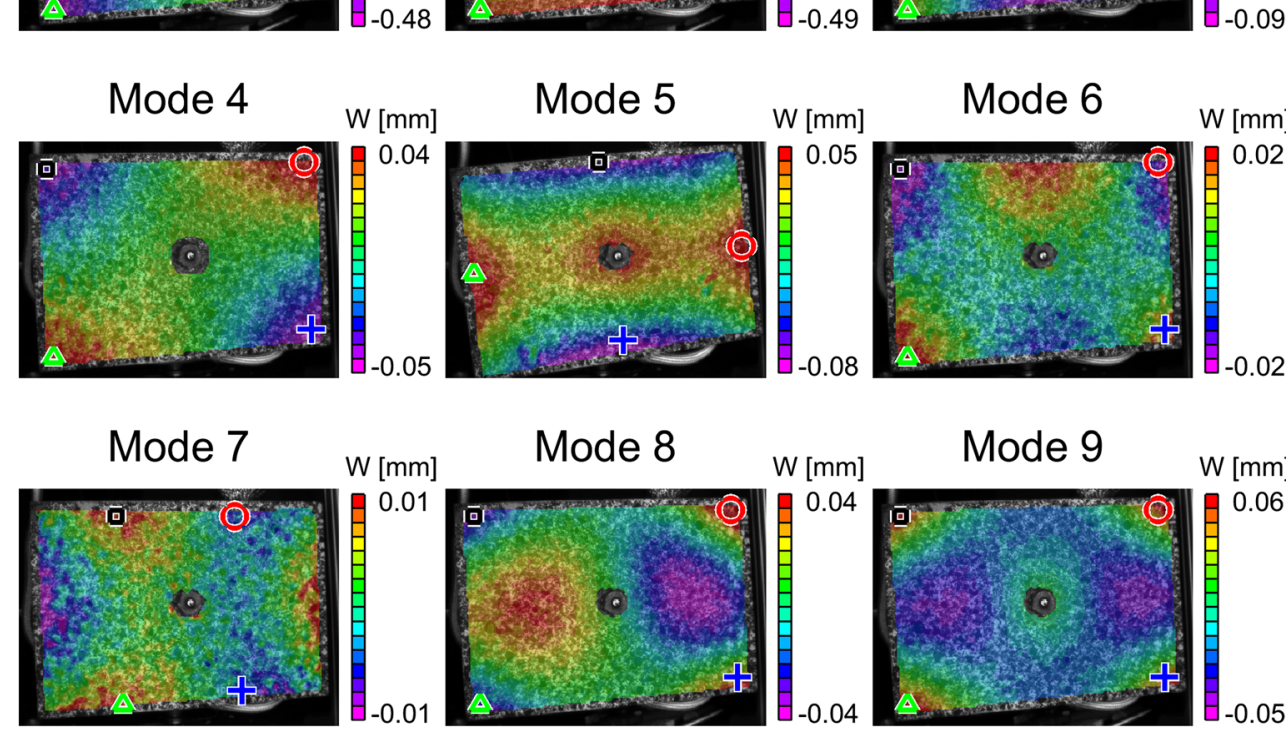

$\mathrm{W}[\mathrm{mm}]$

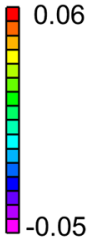

Fig. 8 Out-of-plane

displacements throughout the period of oscillation for the four locations indicated in Fig. 7
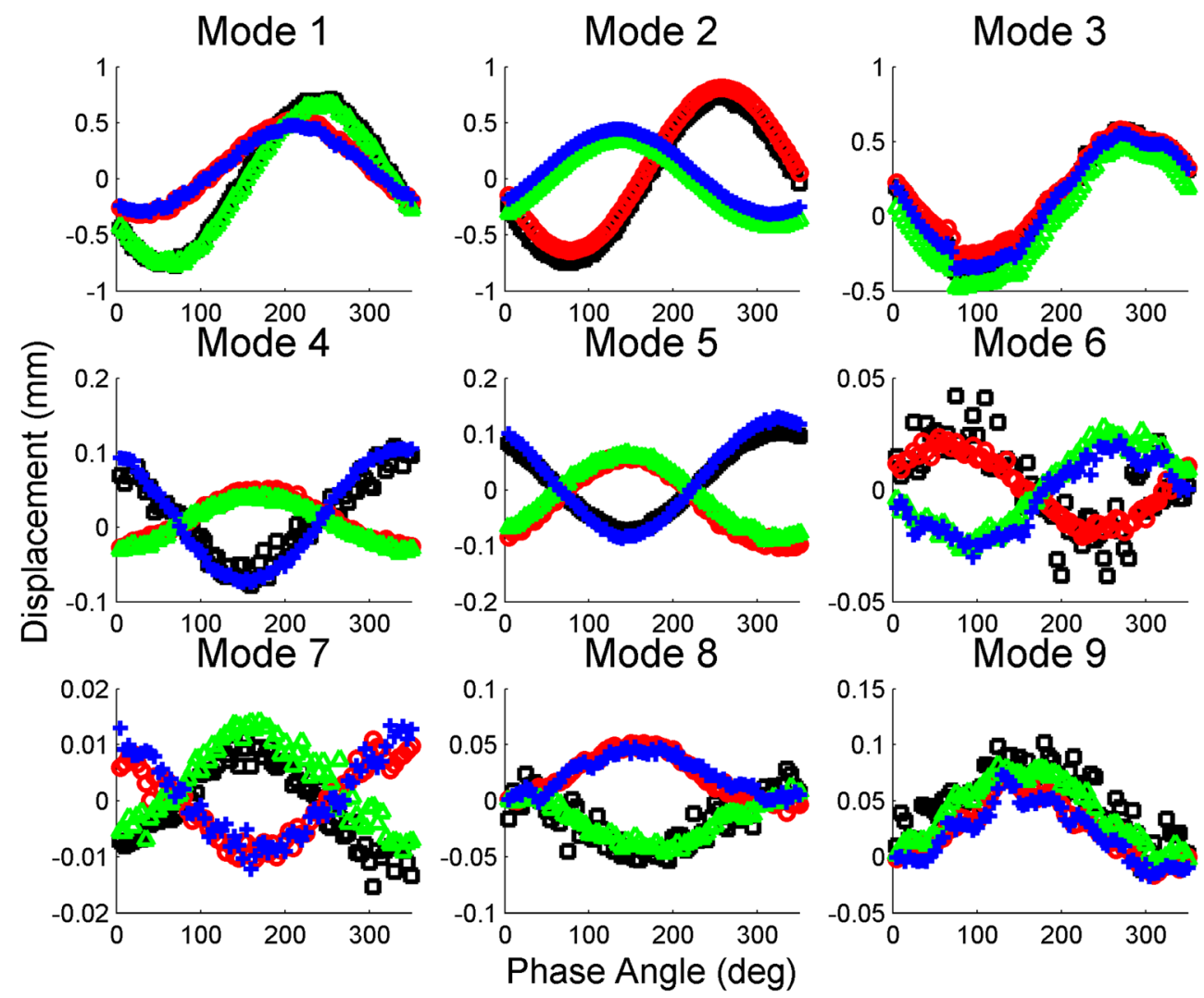
Table 1 Resonant frequencies $(\mathrm{Hz})$ of mode shapes at room temperature (RT) and high temperature (HT)

\begin{tabular}{lllll}
\hline & RT DIC (Fig. 7) & HT DIC (Fig. 9) & RT FEA (Fig. 10) & HT FEA (Fig. 11) \\
\hline Mode 1 & 104 & 107 & 141 & 144 \\
Mode 2 & 140 & 139 & 213 & 217 \\
Mode 3 & 314 & 273 & 214 & 218 \\
Mode 4 & 385 & 295 & 316 & 321 \\
Mode 5 & 735 & 665 & 560 & 574 \\
Mode 6 & 879 & 690 & 794 & 808 \\
Mode 7 & 1080 & 787 & 925 & 941 \\
Mode 8 & 1164 & 1173 & 1190 & 1170 \\
Mode 9 & 1325 & 1251 & 1170 & 1150 \\
\hline
\end{tabular}

maximum and minimum locations seen in Fig. 8, although as frequency increases the measurements become noisier. Note that the resonant frequencies of these modes are provided in Table 1 .

The modal shapes measured by DIC for the estimated temperature distribution in Fig. 4 are plotted in Fig. 9. With only a few small differences, the mode shapes are similar to those in Fig. 7 for the room temperature excitation. Most notably for Mode 8, the left and right halves of the plate are antisymmetric at room temperature but are no longer antisymmetric at high temperature.

The corresponding modal shapes obtained from the simulations are plotted for room temperature in Fig. 10 and for high temperature in Fig. 11. Each simulation reproduced all nine of the modes which were observed experimentally, although at somewhat different frequencies. The resonant frequencies observed at both room temperature and at high temperature, for both DIC and FEA, are summarized in Table 1 and plotted in Fig. 12. Generally as temperature increased, the experimentally measured frequencies for each mode tended to decrease by between 6 and $27 \%$, with the exception of modes 1 and 2, which both represent rigid-body motion and their frequencies stayed roughly the same. One possible explanation for the decrease in frequency is that as temperature increases, the elastic modulus of the material decreases, allowing resonant deformation to occur at lower frequencies. Since rigid-body motion does not involve deformation the change in modulus is irrelevant, and consequently the frequencies of the rigid-body modes remain the same. Other researchers have observed up to an $8 \%$ decrease in natural or resonant frequency for free-
Fig. 9 Modal shapes obtained from stereo-DIC for the temperature distribution in Fig. 4, plotted overtop of the photo in Fig. 1
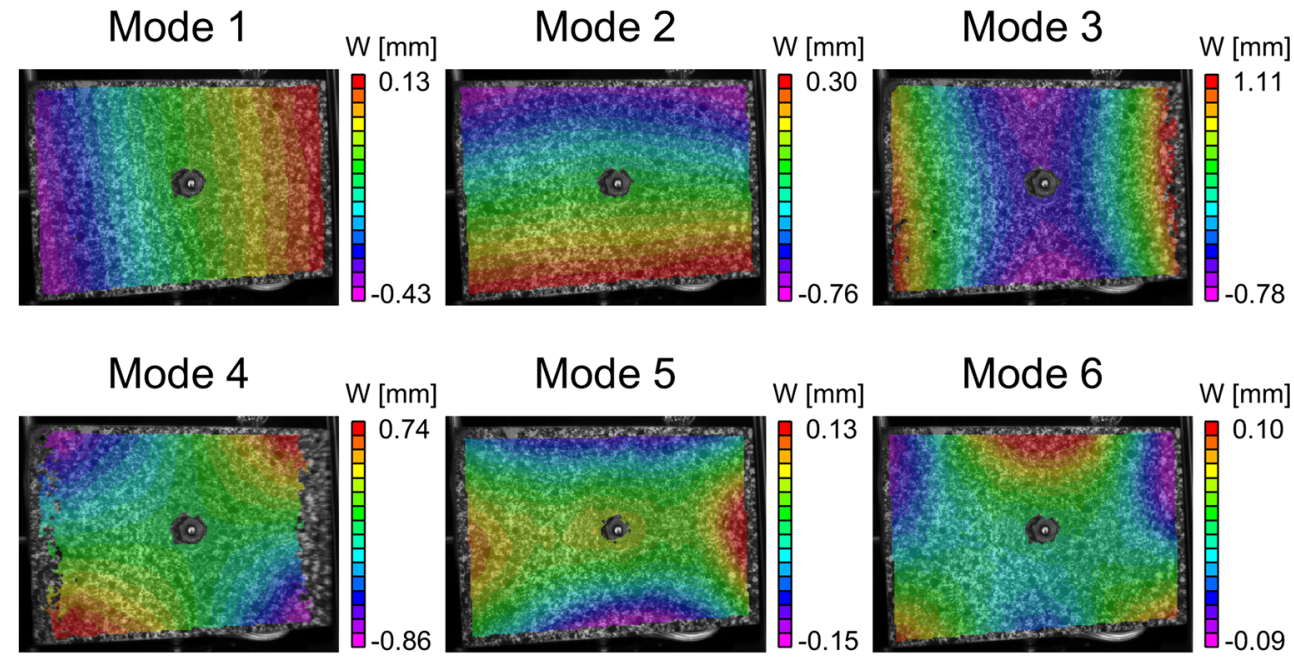

Mode 7
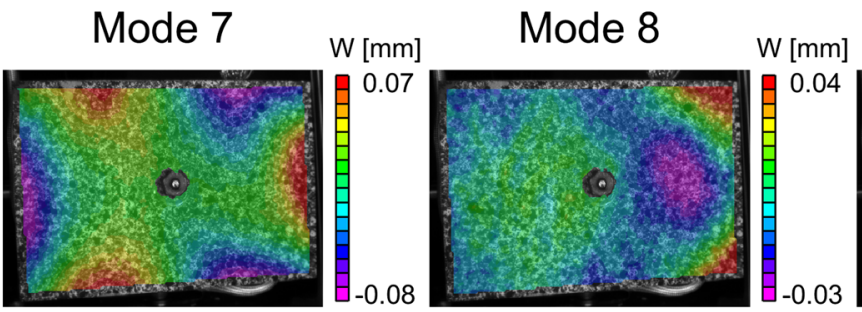

Mode 9

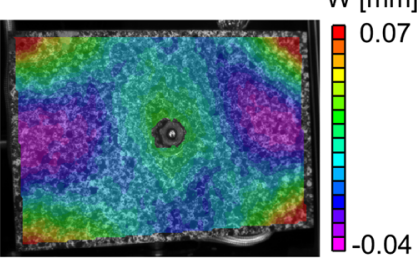




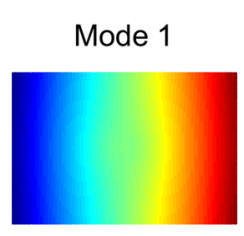

Mode 4

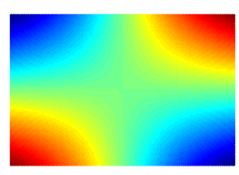

Mode 7
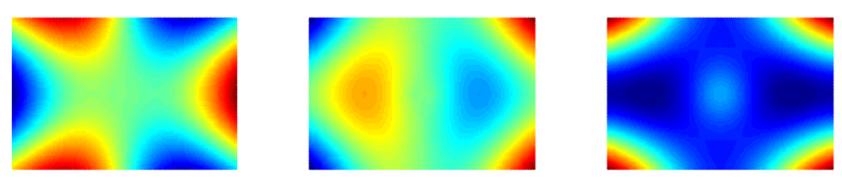

Fig. 10 Modal shapes obtained from the finite element analysis at room temperature

free aluminum plates which are thermally loaded rapidly at $70^{\circ} \mathrm{C} / \mathrm{sec}$ [14]. Another possible mechanism for the changes in frequencies with temperature is the occurrence of buckling due to the uneven heating of the plate. Mead [15] performed simulations in which the center of a rectangular plate was heated more than the edges causing the center of the plate to expand more than the edges, which formed a stabilizing ring. Mead observed that as overall temperature increased, the resonant frequencies changed with temperature and some eventually vanished as the temperature reached a critical value.

There are no significant changes in the simulated frequencies with the change in temperature. One possible explanation is that the model used in the simulation was too simplistic.

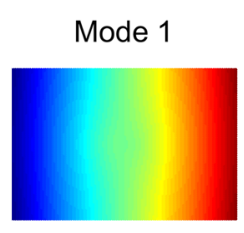

Mode 4

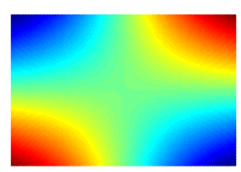

Mode 7

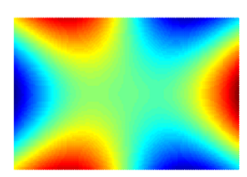

Fig. 11 Modal shapes obtained from the finite element analysis at the temperatures in Fig. 6

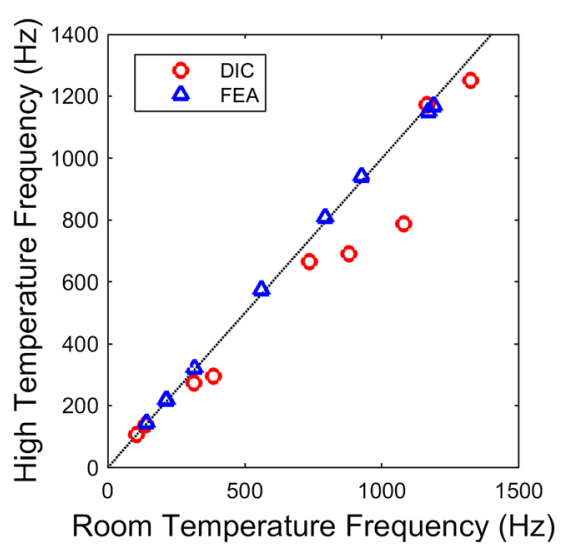

Fig. 12 Comparison of the resonant frequencies in Table 1 observed at room temperature (horizontal axis) and high temperature (vertical axis)

Although the material properties in each element were informed by the temperature distribution, the discrepancy in frequencies might also be due to stresses caused by uneven heating which were not included in the model. Another explanation might be that temperatures in the plate fluctuated as the distance between the induction coil and the plate changed throughout the period of oscillation, but these fluctuations were neither detected experimentally nor included in the simulation. Nonetheless, the model does capture well the main features of the nine modes.

\section{Image Decomposition Analysis}

The simulated mode shapes are compared quantitatively to the experimentally measured mode shapes using a recently developed image decomposition technique [16, 17]. In brief, the technique works by using a set of polynomials to describe the modal shapes such that a knowledge of the polynomials used and their coefficients provides a complete description of the modal shape. In this work, two-dimensional Tchebichef polynomials were used with 55 terms to ensure a high-fidelity representation of the modal shapes; however, in most cases the contribution from the higher order terms was small or negligible with the first fifteen terms dominating. The kernels for these fifteen terms are shown in Fig. 13. The value of the coefficient corresponding to each kernel can be considered to represent the contribution of that kernel to representing the modal shape. A summation of the contribution from the kernels allows the modal shape to be reconstructed. The error in the decomposition process can be assessed by comparing the original and reconstructed data and calculating the average squared residual for the images, $u^{2}$. This specific image decomposition technique is helpful because rather than comparing the behavior of every Gauss integration point in the simulations with DIC correlation points individually, the mode shapes can be compared in terms of fewer parameters, i.e., the Tchebichef coefficients. These are also more physically 
Fig. 13 First 15 Tchebichef

kernels

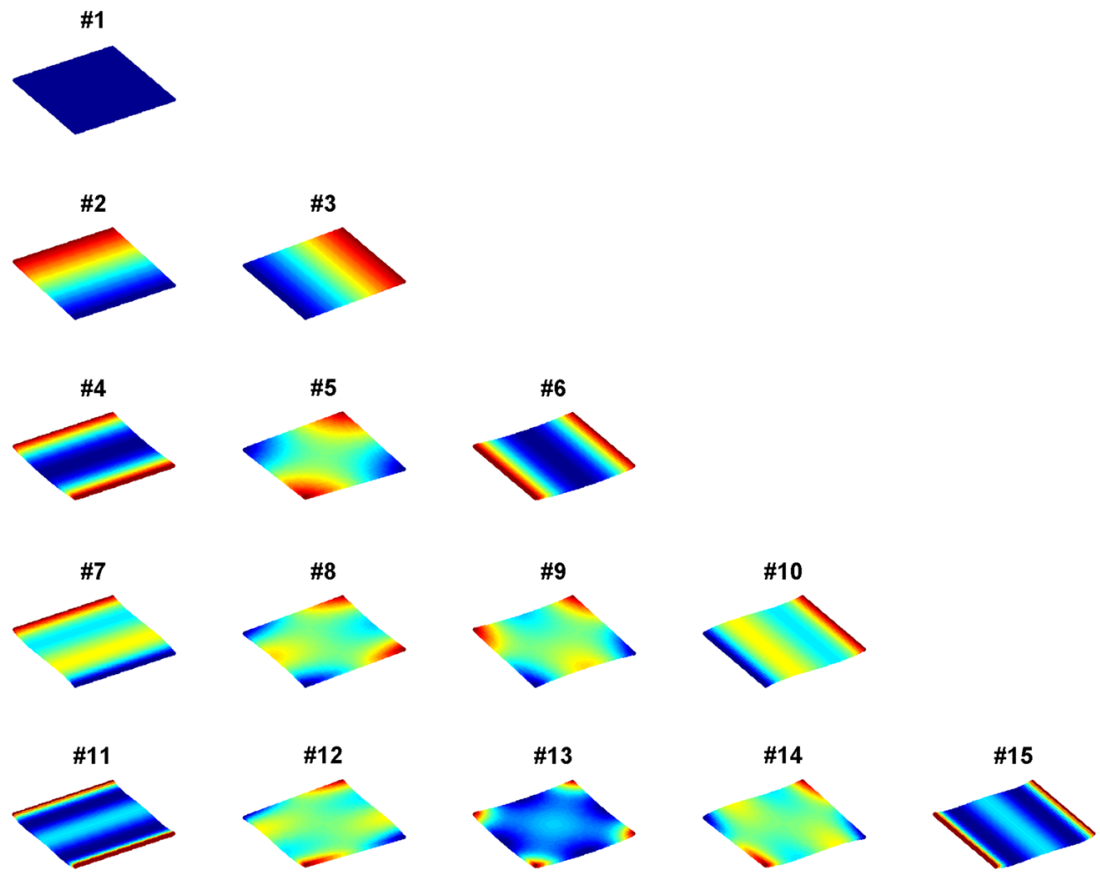

relevant because, as can be seen in Fig. 13, the Tchebichef kernels can be related to specific plate deformations, e.g., kernels 2 and 3 represent plate tilt, kernels 4 and 6 bending, etc.

The Tchebichef decomposition and reconstruction process is illustrated in Fig. 14, using DIC data for Mode 6 at high temperature as an example. In step 1, the DIC data is rotated and mapped onto a square domain. Such a transformation is necessary because the plate had a slight in-plane rotation relative to the cameras which can be seen in Fig. 7. To perform the rotation, the coordinates of the two top corners of the plate are compared to find the small angle, $\theta$ between them relative to the $\mathrm{x}$-axis of the DIC coordinate system, defined by

$\theta=\tan ^{-1}\left(\frac{y_{2}-y_{1}}{x_{2}-x_{1}}\right)$

where $\left(x_{2}, y_{2}\right)$ are the coordinates for the top right corner of the plate, and $\left(x_{1}, y_{1}\right)$ are the coordinates for the top left corner of the plate. The coordinates of all experimental data points were mapped by applying the small rotation about the center of the plate as follows:

$$
\begin{aligned}
& x^{\prime}=\left(x-x_{o}\right) \cos (\theta)-\left(y-y_{o}\right) \sin (\theta) \\
& y^{\prime}=\left(x-x_{o}\right) \sin (\theta)+\left(y-y_{o}\right) \cos (\theta)
\end{aligned}
$$

where $\left(\mathrm{x}^{\prime}, \mathrm{y}^{\prime}\right)$ are the coordinates of the plate in the rotated coordinate system, $(x, y)$ are the coordinates in the initial system, $\left(x_{o}, y_{o}\right)$ are the coordinates for the center of the plate. Once the rotation is completed, the coordinates are then mapped onto a normalized square domain by dividing all $x$-coordinates by the width of the plate, and dividing all $y$-coordinates by the height. Note that a hole in the data is still present at the center of the plate where the attachment nut was excluded from the DIC analysis. The magnitudes of the data were also normalized such that all displacements were plotted from -1 (purple in Figs. 7 and 9) to +1 (red).

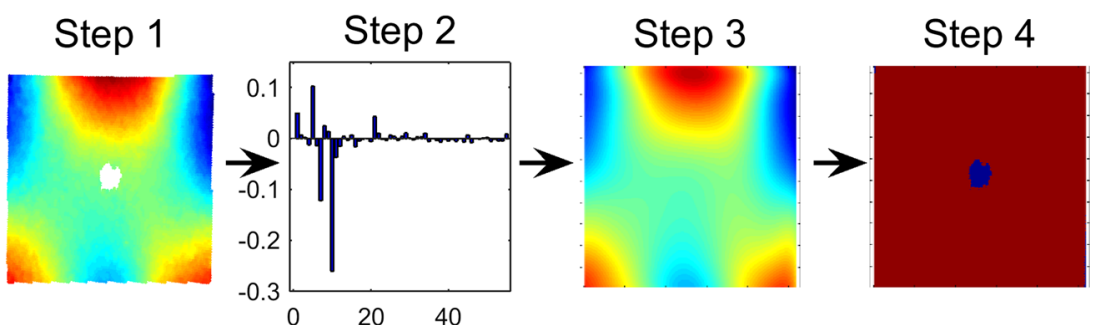

Fig. 14 Illustration of the Tchebichef decomposition process, using DIC data for Mode 6 at high temperature (see Fig. 9): in step 1 the oblique view of the rectangular plate is transformed into perpendicular view of a square; in step 2 the data are decomposed using Tchebichef polynomials and the barchart shows the values of the corresponding coefficients; in step 3 the coefficients are used to reconstruct the data to ensure they provide a high-fidelity representation; and in step 4 the pixels with residuals less than $3 \mathrm{u}$ are plotted in red while the pixels with residuals greater than $3 \mathrm{u}$ are plotted in blue 
In step 2, a decomposition algorithm computes the Tchebichef coefficients which are shown in the bar chart [16-18]. In step 3, the shape is reconstructed by taking the summation of each kernel multiplied the coefficients computed in step 2. The reconstructed data, which contain no hole for the attachment nut, are then compared to the initial data to assess the error produced by the decomposition, in terms of the average squared residual, $u^{2}$

$u^{2}=\frac{1}{N} \sum_{i, j}^{N}(\hat{I}(i, j)-I(i, i))^{2}$

where $\hat{I}(i, j)$ is the reconstructed value of the original image, $I(i, j)$. In step 4 , it is ensured that no location shows a clustering of residuals greater than $3 u$ where a cluster is defined as a group of adjacent pixels comprising $0.4 \%$ or more of $N$, the total number of pixels in the region of interest [18]. Consequently, the error in the reconstruction is expected to be less than $3 u$, except for the pixels corresponding to the hole in the data. In Fig. 14, the pixels with residuals less than $3 u$ are plotted in red and the pixels greater than $3 u$, corresponding only to the hole in the data, are plotted in blue.

In this study, the average residual, $u_{\text {resid }}$ varied from 0.034 to 0.138 for the room temperature experimental results and from 0.027 to 0.071 for the high temperature results. The total uncertainty in the Tchebichef feature vector, $u\left(S_{E}\right)$, is defined by combining the average residual from the reconstruction process with the uncertainty in the experimental setup, $u_{\text {meas }}$ as follows:

$u\left(S_{E}\right)=\sqrt{u_{\text {meas }}^{2}+u_{\text {resid }}^{2}}$

For this study, the results from a previous dynamic assessment of a high-speed DIC system [18] were used as typical values for $u_{\text {meas }}$. Relative uncertainty of $1.8 \%$ of the total measurement range was used, which resulted in a value of $u_{\text {meas }}=0.018$

The Tchebichef coefficients representing each experimentally measured mode shape are plotted against those for each corresponding simulated mode shape for room temperature in Fig. 15 and for high temperature in Fig. 16. The coefficient of the first kernel, which corresponds to a rigid out-of-plane translation, as shown in Fig. 13, and is unrelated to the deformation of the plate, is excluded from the figures. The remaining 54 coefficients are all included, although in reality most of the mode shapes can be reconstructed using relatively few coefficients or kernels. The coefficients with larger magnitudes are farther away from the plot origin and hence represent those kernels that contribute most strongly to the modal shape. The largest coefficients are labeled with the numbers in parentheses corresponding to the kernels in Fig. 13.

Each plot also includes a dashed line of gradient one, which is indicative of perfect agreement between the simulation and the experimental results. The closer a data point lies to this line, the better the agreement is for that coefficient between simulation and experiment $[16,18]$. In the comparison, it is likely that there will be noise in the experimental data and errors in the simulation which will cause the points to deviate from this line. Therefore an acceptable area can be defined around the ideal line as $+/-2 u\left(S_{E}\right)$. If all of the data points from the comparison of the Tchebichef feature vectors fall within this area then the simulation can be deemed an acceptable representation of the experiment [18].

In general, the uncertainty bands are smaller for the high temperature results (Fig. 15) than for the room temperature results (Fig. 16). This is a result of a lower average residual in the reconstruction of the high temperature experimental results because the larger displacements have less noise. For the majority of the plots, all of the data points fall within the acceptable area, and many of the data points fall on the ideal line, indicating agreement between the simulation and the experiment. For example for Mode 3 in Fig. 15, only one Tchebichef kernel is dominant - specifically \#6 in Fig. $13-$ which corresponds to a shape produced by bending about the vertical axis. Most other kernels have coefficients clustered at the origin of the plot in Fig. 15, i.e., they are near zero. Overall Figs. 15 and 16 show that there is a very good comparison of the spatial distribution of out-of-plane displacements between the predicted and measured resonant modes, although as was seen in Table 1 the corresponding frequencies are not predicted as closely. One notable exception to this is mode 3 at high temperature. In this mode there is a kernel, $\# 2$, which falls outside the acceptable area defined by $+/-2 u\left(S_{E}\right)$. This kernel has a significant non-zero value for the simulation data but is zero-valued for the DIC data. Kernel \#2 corresponds to a rigidbody out-of-plane rotation about a horizontal axis, and is the dominant kernel in mode 2 . The simulation predicts mode 2 and mode 3 to occur very close to each other in the frequency domain, i.e. 217 and $218 \mathrm{~Hz}$, respectively, and hence perhaps there is significant interaction between these modes in the model, which is not seen in the data from experiment when the gap between the modes in the frequency domain is 174 and $134 \mathrm{~Hz}$ respectively for the room and high temperature.

There was some concern that support conditions, i.e., the connections between the stinger and plate could be responsible for some of the discrepancies observed between the measured and predicted results. The effect of the length and stiffness of stinger were explored and found to change the natural frequencies but to have no effect on the resulting displacement fields.

The data presented in Figs. 7, 8, 9, 10, 11, 12, 13, 14 and 15 is summarized in Fig. 17. Each mode is represented by a row in the figure in which those Tchebichef coefficients that were more than $20 \%$ of the value for the largest coefficient for each mode shape have been plotted as bars under the corresponding column for the Tchebichef kernel. Data from both experiment (red) and simulation (blue) have been included as well as at 
Fig. 15 Comparison of Tchebichef coefficients representing the data obtained from experiment (horizontal axis) and simulation (vertical axis) at room temperature. Kernels which have significantly large coefficients are indicated by their kernel number in parentheses

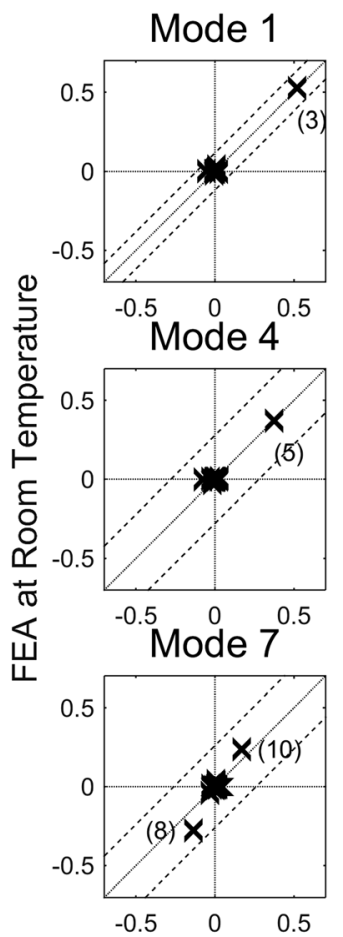

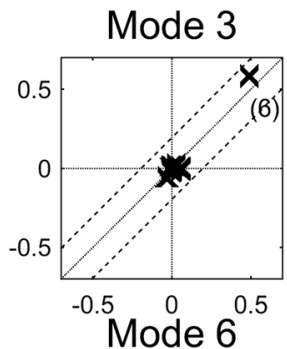
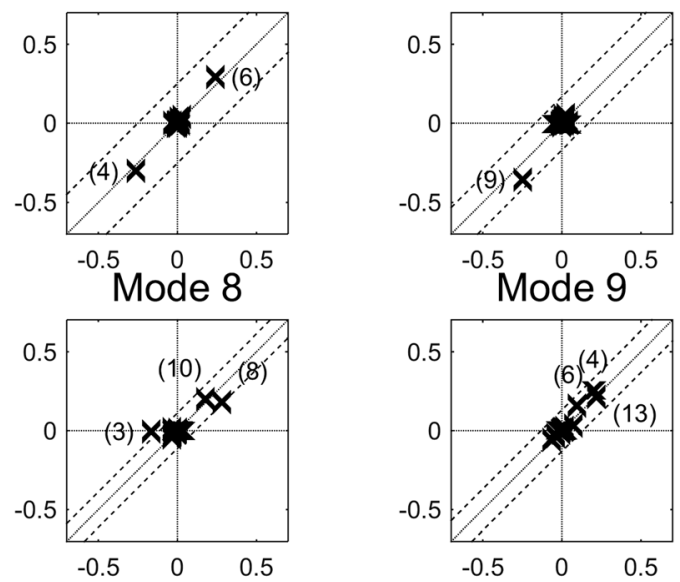

DIC at Room Temperature

room (dark colors) and high (bright colors) temperature. The exclusion of low value coefficients following the approach of Lampeas et al. [19] simplifies the figure without excluding any significant information. The shape represented by the kernels is shown along the top of the figure. A comparison of the height of the bars in each chart provides information both about the changes in behavior from room to high temperature (left pair for experiment or right pair for simulation) and about the degree to which the simulation represents the experiment (right pair relative to left pair). The column in which the larger
Fig. 16 Comparison of Tchebichef coefficients representing the data obtained from experiment (horizontal axis) and simulation (vertical axis) for the temperature distribution shown in Fig. 3

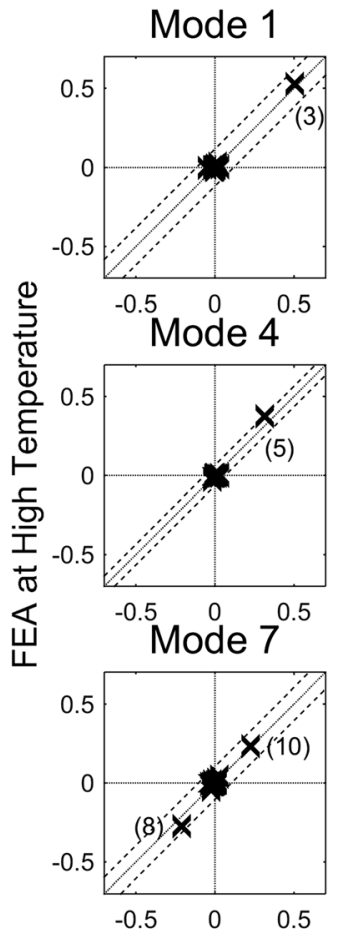

Mode 3
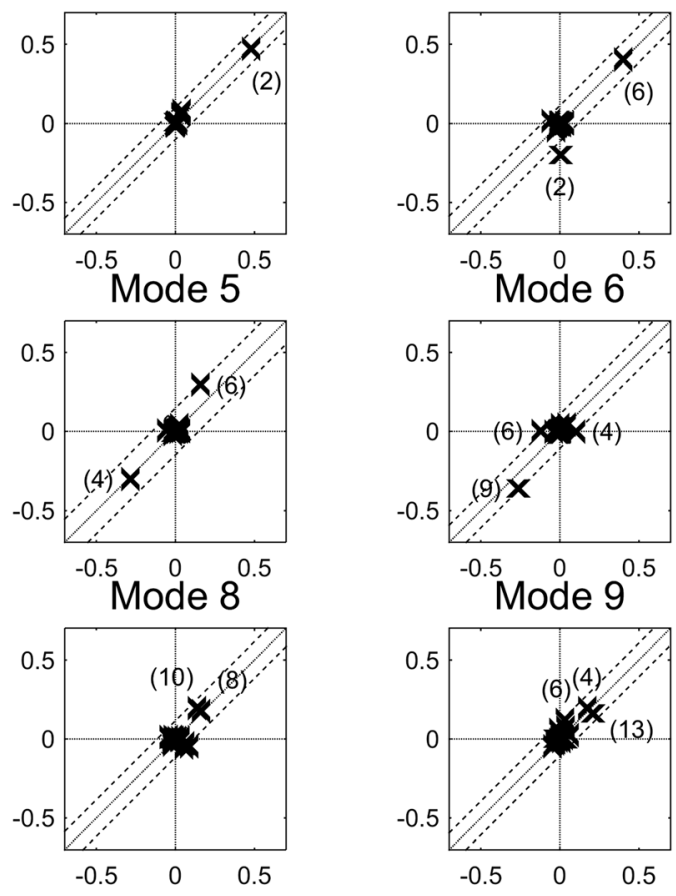

DIC at High Temperature 
Fig. 17 A schematic diagram summarizing the results with the Tchebichef kernels shown along the top row with the corresponding coefficients from both room (darker color) and high (bright color) temperature tests shown in the bar charts from both the experiment (red) and simulation (blue)

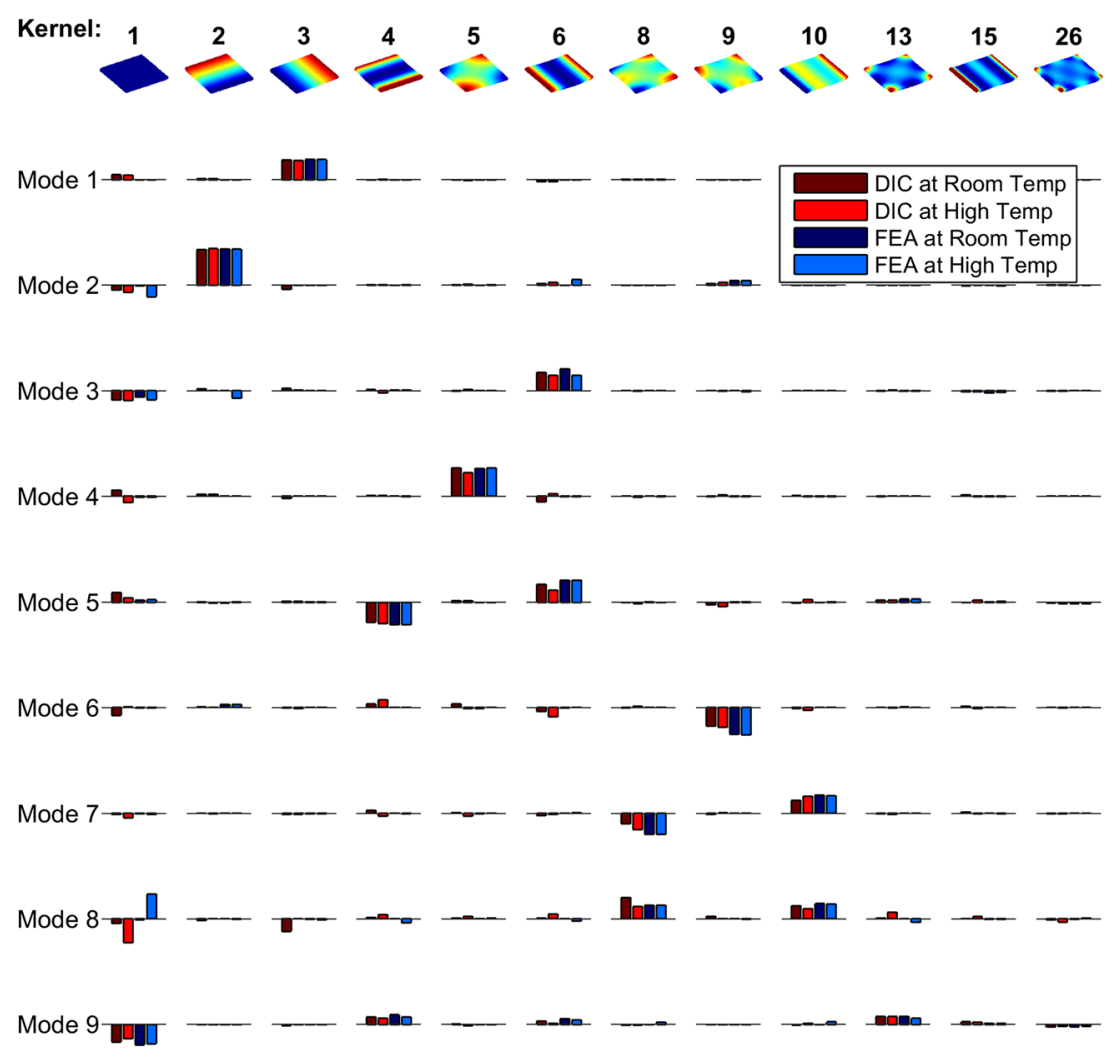

bars appear for a particular mode provide an indication of the dominant components of the deformation. Note that negative values simply indicate that the out-of-plane deformation was in the opposite orientation to the Tchebichef kernels. So, for instance examining mode 3 again, it can be seen that the simulation reproduces the decrease in the sixth coefficient with increasing temperature observed in the experiment but predicts a significant value for the second coefficient which is not present in the experiment. The dominant role of the second coefficient in mode 2 can also be observed. When the first coefficient is ignored, which represents the 'piston term' or average magnitude, the figure shows a trend in which the higher magnitude bars are found along the diagonal from top left to bottom right, i.e. the higher order, more complex kernels are used to represent the higher order modes, which is entirely expected. The higher order modes also tend to require more kernels to represent them, which is again expected due to their more complex shape. Overall, the figure supports the conclusion that the simulation is a good representation of the experiment and permits the more complex modal shapes to be visualized in terms of the simpler kernel shapes.

\section{Conclusions}

The work described here has combined, for the first time, high frequency vibratory loading (up to about $1300 \mathrm{~Hz}$ ) at elevated temperature (up to $600{ }^{\circ} \mathrm{C}$ ) with the full-field optical diagnostics of stereo-DIC. This combination has provided high quality experimental data suitable for the validation of numerical results. Using this combined loading experimental set-up with induction heating and shaker-induced vibration, the thermoacoustic behavior of a rectangular plate of Hastelloy-X was explored. By investigating the first 9 resonant modes at both room temperature and high temperature, and measuring mode shapes for each of the resonant frequencies using stereo-DIC, the influence of temperature on the thermomechanical vibratory response of the plate was assessed. A decrease in resonant frequencies was seen with increasing temperature, although the first 9 mode shapes themselves were similar. Companion finite element numerical simulations that accounted for the temperature dependence of elastic modulus also produced very similar mode shapes, although the influence of temperature on modal frequencies was not as significant as observed experimentally. As a model process for validating simulation results based on this type of experimental data collection an image decomposition technique based on 2D Tchebichef polynomials [18] was used. The image decomposition, which allows comparison between numerics and experiments using the fitted Tchebichef coefficients rather than the entire images, confirmed quantitatively the qualitative observation that as temperature increased the resonant mode shapes remained very similar. Overall, the results of this combined thermalmechanical study point to the conclusion that the simulation 
is a good representation of the experiment both at room temperature and at high temperature (in fact a little better at high temperature as the signal to noise ratio in that experiments is larger). Further study would include a continuous recording of data rather than just at resonant frequencies, so that the transition from one mode to the other may be better studied. Since the DIC technique is not material or specimen dependent, it should be relatively simple to apply these methods to other scenarios involved thermo-vibratory excitation.

Acknowledgments This effort was sponsored by the Air Force Office of Scientific Research, Air Force Material Command, USAF under grant numbers FA8655-11-3083 and FA9550-12-1-0386. The U.S. Government is authorized to reproduce and distribute reprints of Governmental purpose notwithstanding any copyright notation thereon. Major Matt Synder (EOARD) and Dr. David Stargel (AFOSR) respectively are the program officers for these grants. EAP is the recipient of a Royal Society Wolfson Research Merit Award. The collaboration that was central to this study was supported by a Royal Academy of Engineering Distinguished Visiting Fellowship awarded to JL.

Open Access This article is distributed under the terms of the Creative Commons Attribution 4.0 International License (http:// creativecommons.org/licenses/by/4.0/), which permits unrestricted use, distribution, and reproduction in any medium, provided you give appropriate credit to the original author(s) and the source, provide a link to the Creative Commons license, and indicate if changes were made.

\section{References}

1. Grant BMB, Stone HJ, Withers PJ, Preuss M (2009) Hightemperature strain field measurement using digital image correlation. J Strain Anal Eng Des 44(4):263-271

2. Novak MD, Zok FW (2011) High-temperature materials testing with full-field strain measurement: experimental design and practice. Rev Sci Instrum 82(11):115101

3. Chen X, Xu N, Yang L, Xiang D (2012) High temperature displacement and strain measurement using a monochromatic light illuminated stereo digital image correlation system. Meas Sci Technol 23(12): 125603

4. Pan B, Wu D, Wang Z, Xia Y (2011) High-temperature digital image correlation method for full-field deformation measurement at $1200{ }^{\circ} \mathrm{C}$. Meas Sci Technol 22(1):015701
5. Hammer JT, Seidt JD, Gilat A (2014) Strain measurement at temperatures up to $800{ }^{\circ} \mathrm{C}$ utilizing digital image correlation. In: Jin $\mathrm{H}$, Sciammarella C, Yoshida S, Lamberti L (eds) Advancement of optical methods in experimental mechanics, vol 3. Springer International Publishing, New York, pp 167-170

6. Berke RB, Lambros J (2014) Ultraviolet digital image correlation (UV-DIC) for high temperature applications. Rev Sci Instrum 85(4):045121

7. Sha YD, Wei J, Gao ZJ, Zhong HJ (2014) Nonlinear response with snap-through and fatigue life prediction for panels to thermoacoustic loadings. J Vib Control 20(5):679-697

8. Abotula S, Heeder N, Chona R, Shukla A (2014) Dynamic thermomechanical response of hastelloy $\mathrm{X}$ to shock wave loading. Exp Mech 54(2):279-291

9. Vautrot M, Balland P, Hopperstad OS, Tabourot L, Raujol-Veillé J, Toussaint F (2014) Experimental technique to characterize the plastic behaviour of metallic materials in a wide range of temperatures and strain rates: application to a high-carbon steel. Exp Mech 54(7): $1163-1175$

10. Zinn S, Semiatin SL (1988) Induction coil design and fabrication tech note - part 1. Heat Treating Magazine

11. Haynes International, Inc. HASTELLOY ${ }^{\circledR} X$ Alloy. [Online]. Available: http://www.haynesintl.com/XHASTELLOYALLOY.htm. Accessed: 03-Nov-2014

12. Hack E, Lin X, Patterson EA, Sebastian CM (2015) A reference material for establishing uncertainties in full-field displacement measurements. Meas Sci Technol 26:075004

13. Altair H (2014) RADIOSS, and Optistruct. Altair, Troy

14. Jeon B, Kang H, Lee Y (2011) Free vibration characteristics od rectangular plate under rapid thermal loading. Presented at the 9th Int'l. Conf. on Thermal Stress, Budapest, Hungary

15. Mead DJ (2003) Vibration and buckling of flat free-free plates under non-uniform in-plane thermal stresses. J Sound Vib 260(1): $141-165$

16. Wang W, Mottershead JE, Ihle A, Siebert T, Reinhard Schubach H (2011) Finite element model updating from full-field vibration measurement using digital image correlation. J Sound Vib 330(8): $1599-1620$

17. Sebastian C, Hack E, Patterson E (2013) An approach to the validation of computational solid mechanics models for strain analysis. J Strain Anal Eng Des 48(1):36-47

18. CWA16799 (2014) Validation of computational solid mechanics models. Comite Europeen de Normalisation, Brussels

19. Lampeas G, Pasialis VP, Lin X, Patterson EA (2015) On the validation of solid mechanics models using optical measurements and data decomposition. Simul Model Pract Theory 52:92-107 